\title{
JUNO-GROUND-RADIO OBSERVATIONS SUPPORT
}

\author{
B. Cecconi* ${ }^{* \infty}$, P. Zarka*, R. Savelle ${ }^{\dagger}$, P. Le Sidnaer ${ }^{\dagger}$, A. Coffre ${ }^{\ddagger}$ \\ L. Denis ${ }^{\ddagger}$, C. Viou ${ }^{\ddagger}$, A. Konovalenko ${ }^{\S}$, A. Skoryk ${ }^{\S}$, S. Yerin ${ }^{\S}$, \\ Y. Kasaba』, A. Kumamoto", H. Misawaף, T. Tsuchiyaף, Y. Hobara", \\ T. Nakajo**, K. Imai ${ }^{\dagger \dagger}$, V. Ryabov ${ }^{\ddagger \ddagger}$, H. Rothkaehl${ }^{\S}$, G. S. Orton $\mathbb{\mathbb { I }}$

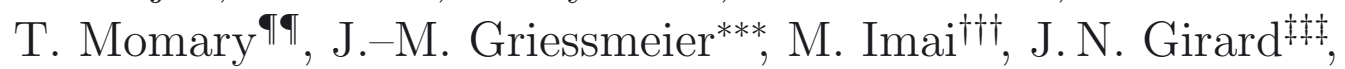 \\ L. Lamy*, M. Anderson ${ }^{\S \S}$, N. André ${ }^{\infty}$, V. Génot ${ }^{\infty}$, R. Ebert ${ }^{\diamond}$,

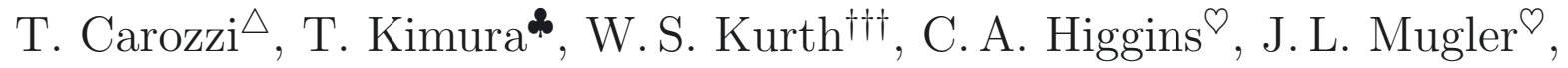 \\ D. Typinsky", T. Clarke ${ }^{\infty}$, J. Sky $\diamond$, R. Flagg ${ }^{\star}$, F. Reyes ${ }^{\star}$, \\ W. Greenman^, J. Brown ${ }^{\star}$, A. Mount», T. Ashcraft», J. Thieman ${ }^{\star} \Delta$,

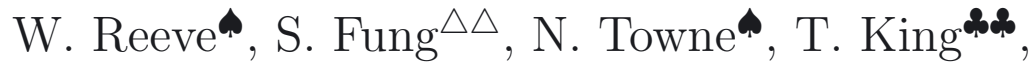 \\ and S. Bolton $\diamond$
}

\begin{abstract}
The JUNO mission is a NASA flagship mission dedicated to the study of Jupiter. Several instruments are dedicated to the study of the Jovian internal magnetic field and its inner magnetosphere. In the frame of the preparation of the NASA/JUNO and ESA/JUICE (Jupiter Icy Moon Explorer) missions on one hand, and the development of a planetary sciences virtual observatory (VO) on the other hand, we are proposing new tools directed to data providers and scientists, in order to ease low frequency radio astronomy data products sharing and discovery. We will focus on

LESIA, Observatoire de Paris, Meudon, France

PADC, Observatoire de Paris/CNRS/PSL, Paris, France

USN, Observatoire de Paris/CNRS/PSL/Université d' Orléans, Nançay, France

I Institute of Radio Astronomy, National Academy of Sciences of the Ukraine, Kharkov, Ukraine

I Tohoku University, Sendai, Japan

" University of Electro-Communications, Tokyo, Japan

** Fukui University of Technology, Fukui, Japan

i† Kochi National College of Technology, Nankoku, Japan

$\ddagger$ Future University, Hakodate, Hakodate, Japan

$\S$ Space Research Center, Polish Academy of Sciences, Warsaw, Poland

II NASA Jet Propulsion Laboratory, Caltech, Pasadena, CA, USA

*** LPC2E, CNRS/Université d' Orléans, Orléans, France

t† Department of Physics and Astronomy, University of Iowa, Iowa City, IA, USA

$\ddagger \ddagger \ddagger$ AIM/IRFU/SAP-CEA, Université Paris Diderot, Saclay, France

$\S \S$ California Institute of Technology, Pasadena, CA, USA
\end{abstract}


ground-based planetary radio observations (thus mainly Jupiter radio emissions), trying for instance to enhance the temporal coverage of Jovian decametric emission. The data service we will be using is EPN-TAP, a planetary science data access protocol developed by Europlanet-H2020-RI/VESPA (Virtual European Solar and Planetary Access). This protocol is derived from IVOA (International Virtual Observatory Alliance) standards. Observations of Jovian radio emissions from the Nançay Decameter Array (France) and the Iitate radio observatory (Japan) are already shared on the planetary science VO using this protocol. Amateur radio data from the RadioJOVE project is also available. We will first introduce the VO tools and concepts of interest for the planetary radioastronomy community. We will then present the various data formats now used for such data services, as well as their associated metadata. We will finally show various prototypical tools that make use of this shared datasets.

$\infty$ CDPP, CNES/CNRS/Université Paul Sabatier/Observatoire de Paris, Toulouse, France

$\diamond$ Space Science Department, Southwest Research Institue, San Antonio, TX, USA

$\triangle$ Institute of Space and Geophysics, Calmers, Onsala, Sweden

* RIKEN Nishina Center for Accelerator-Based Science, Tokyo, Japan

$\checkmark$ Middle Tennessee State University, Murfreesboro, TN, USA

- RadioJOVE, USA

$\infty \infty$ US Naval Research Laboratory, Washington DC, USA

$\diamond \diamond$ Radio-Sky Publishing, USA

$\triangle \triangle$ NASA Goddard Space Flight Center, Greenbelt, MD, USA

क4 IGPP, UCLA, Los Angeles, CA, USA 